\title{
Lipoprotein (a) and Comprehensive Lipid Tetrad Index in Young Patients of Coronary Artery Disease
}

\author{
Giri S, Rajagopalan B, Dwivedi S, Srivastva DK, *Singh s, Kaur I, \\ Objective:
}

To compare the clinico-biochemical profile of young patients ( $\leq 40$ years) having coronary artery disease $(C A D)$ with age-sex matched controls with a special reference to lipoprotein (a) $[\mathrm{Lp}(\mathrm{a})]$ and comprehensive lipid tetrad index (CLTI).

\section{Materials:}

Forty seven consecutive patients of $\leq 40$ years of age diagnosed as CAD at GTB Hospital, Delhi were evaluated for risk factors like smoking, hypertension, diabetes, family history of CAD, body mass index (BMI), waist-hip ratio and lipid profile including $L p(a)$. CLTI is calculated by a formula suggested by EA Enas $[(C L T]=\{T C \times T G \times L p(a)\} / H D L-C)]$. These parameters were compared with age and sex matched controls (49) without CAD.

\section{Result:}

The cases and controls included equal number of females and diabetics. The BMI and waist-hip ratios were statistically comparable in both groups. Number of smokers was more among cases $38(76.6 \%) \mathrm{v} / \mathrm{s}$ controls 28 (58.1\%). Family history of smoking was moderately significant in cases. Mean total cholesterol and triglyceride in cases and controls were $182.5 \sim 50.6$ vs. $162.0 \pm 39.6 \mathrm{mg} / \mathrm{dl}(p<0.05)$ and $159 \pm 81.1 \mathrm{vs.} 123.0 \pm 32.3 \mathrm{mg} / \mathrm{dl}(p<0.008)$ respectively. Total cholesterol and triglycerides were raised in cases than controls. In both groups mean LDL cholesterol was with in normal range. The mean levels of HDL cholesterol were lower than normal limit in both the groups (as per ATP III guidelines). The LDL and HDL cholesterol were statistically comparable among cases and controls respectively (see table). 


\section{Table: Levels of various components of Lipid profile in cases and controls,}

\begin{tabular}{|l|l|l|l|l|}
\hline Variable (mg/dl) & $\begin{array}{l}\text { GP: Cases mean } \\
\pm \text { SD }\end{array}$ & $\begin{array}{l}\text { GP: Controls mean } \\
\mathbf{\pm S D}\end{array}$ & P-Value & Sign \\
\hline Serum Lp (a) & $13.23 \pm 7.89$ & $6.28 \pm 3.47$ & $<0.0001$ & $\mathrm{HS}$ \\
\hline Total Cholesterol & $182.5 \pm 50.6$ & $162.0 \pm 39.6$ & $<0.05$ & $\mathrm{~S}$ \\
\hline LDL Cholesterol & $115.49 \pm 43.51$ & $112.88 \pm 28.87$ & $>0.7$ & $\mathrm{NS}$ \\
\hline HDL Cholesterol & $38.55 \pm 11.02$ & $39.71 \pm 6.38$ & $>0.5$ & $\mathrm{NS}$ \\
\hline Triglycerides & $159.0 \pm 81.1$ & $123.0 \pm 32.3$ & $<0.008$ & $\mathrm{~S}$ \\
\hline CLTI & $10886.71 \pm 9037.3$ & $\pm 3397.62 \pm 2892.5$ & $<0.0001$ & $\mathrm{HS}$ \\
\hline
\end{tabular}

Although in our laboratory, we did not observe very high values of $L p(a)$ neither among cases nor in controls, still the mean $L p(a)$ values were significantly higher in cases than controls $(p<0.0001)$. The distribution of $L p(a)$ amang cases was in higher range than controls, On logistic regression the Lo(a) has shown association with young CAD $(p<0.002$, odds ratio 1.234$)$.

In this study, we also observed the higher mean CLTI values in cases than controls $(<0,0001)$, Although mean LDL cholesterol (a potent atherogenic lipoprotein) was with in normal range and comparable in both groups, however, CLTI was very high in patients having CAD than controls. Here we also found lower HDL cholesterol levels in our population with and without CAD.

\section{Conclusion:}

The higher values of $L p(a)$ and CLTI observed in this study showed a positive correlation to CAD in young patients. We observed normal LDL oholesterol; how ever, HDL cholesterol was lower than normal limit in young population studied.

University College Medical Sciences \& GTB Hospital, Delhi, 'Central Drug Research Institute, Lucknow, India, 